\title{
Innovation pédagogique et créativité dans la pratique de l'écrit en classe de FLE
}

\section{Résumé:}

Chez les apprenants en FLE (Français Langue Étrangère), nous constatons une curiosité pour tout ce qui concerne les nouvelles technologies. C'est pourquoi, nous avons intégré l'usage du numérique dans l'enseignement de l'écrit. Nous avons modifié leurs habitudes scripturales: le support sur lequel ils produisent change. Ils utilisent leur ordinateur, tablette, smartphone pour rédiger. Ils choisissent librement le moment où ils produisent et envoient directement leurs écrits à travers un blog. L'enseignant ainsi que l'ensemble du groupe peuvent consulter les productions en dehors des heures habituelles de cours. Nous avons constaté que les écrits numériques diffèrent des écrits sur papier. Les apprenants font preuve de créativité notamment par un travail sur la forme. Les écrits sont partagés en ligne dès que l'enseignant les valide et les apprenants peuvent rebondir sur l'écrit de leurs camarades. Mots clés : autonomie, créativité,projet collectif, écriture numérique, blog

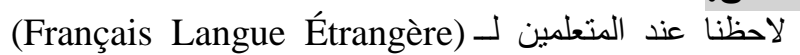
FLE بالتكنولوجيات الجديدة, لذلك أدمجنا استخدام الرقمية فبتدريس الكتابة.

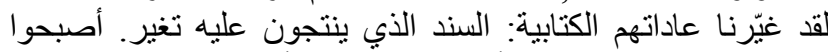

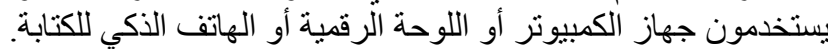

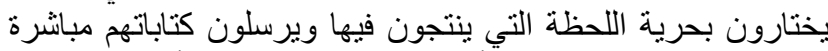

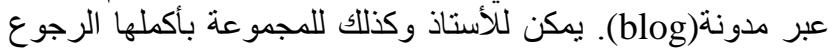

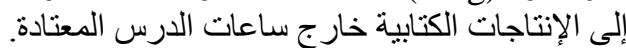

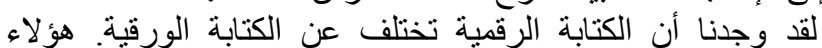

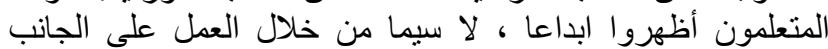

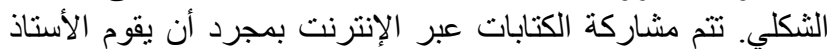

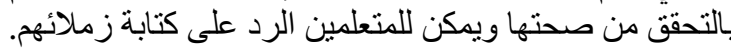

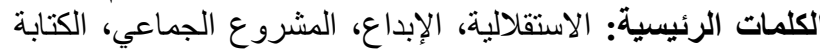

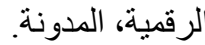

\section{Lobna Raïssi-Djerafi}

École Normale Supérieure de Constantine Laboratoire LANADIF (Laboratoire National de didactique du Français)

\section{Introduction:}

De nos jours, dans un contexte de perpétuel changement, tout enseignant en langue vivante doit ancrer sa pratique dans deux optiques différentes : d'une part le respect des normes établies par le programme de l'université, de l'autre, l'ouverture par la créativité chez les différents protagonistes de la sphère enseignant/ apprentissage. Sans aucune formation, nous avons choisi d'intégrer l'usage du numérique pour la pratique de l'écrit afin de favoriser l'innovation dans les pratiques de cours ainsi que les méthodes d'évaluation. 
Nous avons voulu, par le biais du numérique orienter l'apprenant dans son apprentissage d'une langue étrangère. L'apprenant, friand de nouvelles technologies, a pris l'initiative de s'équiper depuis quelques années de: smartphones, et/ou tablettes, ordinateurs, ce qui lui permet d'avoir accès à internet dans la salle de cours.

L'objet de cette étude exploratoire est d'observer l'impact du numérique dans un module de pratique et technique de l'écrit destiné à des apprenants de FLE. La pratique de l'écrit peut s'avérer particulièrement difficile avec des apprenants peu à l'aise avec la langue française.Nous avons voulu modifier certains dispositifs pédagogiques susceptibles de générer la créativité dans un environnement régulé.

Notre projet visait à améliorer le processus d'apprentissage pour permettre à l'apprenant d'acquérir les notions linguistiques, syntaxiques essentielles à sa formation tout en créant une certaine curiosité envers le savoir grâce à la mise en place d'un projet propice à la créativité. Cette dernière, étant l'essence même de la vie humaine, est à la base de la démarche pédagogique que nous avons voulu mettre en place.

\section{PRÉSENTATION DU CONTEXTE}

Le projet faisant l'objet de notre étude est proposé dans le contexte d'une École Normale Supérieure, pour un public de futurs enseignants du primaire, collège, lycée. Les apprenants sont d'origine sociale et culturelle différente puisque l'École accueille des apprenants de différentes wilayas d'Algérie (ce qui équivaut aux départements en France). Cette richesse socio-culturelle a favorisé l'épanouissement et le développement des apprenants. L'ENS accueille les lauréats du baccalauréat. Elle présente des particularités par rapport aux autres universités :

- La garantie de l'emploi : un poste de professeur permanent au sein de l'Éducation Nationale

- La formation s'articule sur deux volets complémentaires : la théorie et le stage pratique pour les fins de cycle $(\mathrm{Bac}+3, \mathrm{Bac}+4, \mathrm{Bac}+5)$

\section{LE CHOIX DE L'ÉCRITURE NUMÉRIQUE}

Nous n'avons pas reçu de formation spécifique sur l'écriture numérique. En observant les pratiques de nos apprenants, il nous paraissait évident d'introduire les nouvelles technologies afin de leur fournir une meilleure qualité d'apprentissage. Mais ces pratiques restent marginales. Elles ne sont pas encadrées par l'équipe pédagogique et relève de l'initiative personnelle de l'enseignant. Dans notre vie privée et dans notre vie professionnelle, nous avons remarqué que cette génération est friande de nouvelles technologies. Elle acquiert ses connaissances en matière de numérique en autodidacte. Intégrer les 
nouvelles technologies à l'enseignement nous paraissait évident. Nous avons donc pris en compte le profil de nos jeunes apprenants.

Le module pratique de l'écrit englobe deux aspects contrastés : le respect des normes pédagogiques (respect des consignes, du système énonciatif, de la maîtrise du discours, de l'orthographe, de la grammaire, du lexique...) et la volonté de sortir d'une routine souvent source d'ennui aussi bien chez l'apprenant que chez l'enseignant lui-même. La rédaction obéit toujours aux règles de la production écrite (grammaticales, lexicales, ...) mais nous avons essayé d'y introduire une part d'improvisation, d'imagination dans leurs écrits par le biais du numérique. La créativité respecte les acquis précédents qui lui serviront de modèle pour les productions futures. La première nouveauté, concernant le module de production écrite, concerne l'utilisation d'un nouveau support (ordinateur, tablette...) car : « ... ces nouveaux outils constituent un catalyseur qui conduit progressivement l'enseignant à innover au niveau de ses méthodes en les rendant plus centrées sur l'activité de l'apprenant («que vontils faire de ces outils ?»)» (Lebrun, 2007, p. 23).L'enseignant favorise des apprentissages innovants tout en garantissant les progrès des apprenants en FLE. L'activité ne consiste pas à transformer une production format papier en une production électronique avec uniquement un effort fourni sur la mise en page. Il s'agit en réalité de l'association d'une activité réalisée par le biais du numérique et de la mise en commun (que ce soit par la consultation du blog ou lors de la mise en commun en classe) de cette activité en construction.Les apprenants envoient leurs productions via internet. Après une première lecture, l'enseignant les affiche à travers un blog. Les textes y sont placés selon l'ordre d'envoi initial. $^{(1)}$ Cette activité devait leur permettre d'échapper d'une norme pédagogique et de ne plus en rester prisonniers.

Avec l'écriture traditionnelle, nous ne pouvions lire l'ensemble des productions et les corriger en détail lors de la lecture des textes en classe. L'écriture numérique par définition permet le partage de chaque production que ce soit en classe lors de la mise en commun ou individuellement puisque les textes restent accessibles sur le blog. Les apprenants prennent connaissance des écrits et notent les modifications qu'ils envisagent de réaliser lors de la mise en commun. Le groupe est soudé et ouvert aux productions de ses camarades. Le partage des textes que ce soit lorsqu'ils sont publiés sur le blog ou lors des mises en commun en classe encourage les apprenants passifs à participer par l'échange de compétences. Il n'est plus question d'une créativité individuelle seulement mais aussi collective grâce au partage des écrits. Nous écrivons pour être lu et nous cherchons par cette pratique à nous faire comprendre par autrui tout en éveillant leur intérêt.

Pour la troisième année consécutive, nous avons proposé à nos apprenants de fin de cycle $^{(2)}$ le même atelier d'Eva Kavian intitulé « Carnet de voyage » ${ }^{(3)}$ (Kavian, 2009). La proposition d'écriture demande aux apprenants de produire 
sur le thème du voyage, les consignes d'écriture sont les mêmes ${ }^{(4)}$ mais avec quelques modifications. Nous nous sommes également inspirés du travail de Nathalie Portier concernant l'écriture numérique à travers un texte collaboratif, «Une ville sous nos pas ». Il y est question d'une femme qui déambule dans différentes villes. Sa promenade est racontée par des scripteurs anonymes de différents pays qui ne se connaissent pas. C'est cette écriture collective que nous avons tenté de reproduire dans «Carnet de voyage»: raconter la vie d'un apprenant durant son cursus universitaire à partir d'anecdotes appartenant à 46 apprenants. Nous avons entraperçu les potentialités créatives de ce projet à travers un thème qui leur tient à cœur car appartenant à l'intimité de leur vécu alliant ainsi des facteurs cognitifs, des facteurs émotionnels et des facteurs environnementaux. Nous avons opté pour un thème stimulant et assez flexible pour que chaque apprenant puisse y trouver un aspect qui l'intéresse et pour qu'il puisse laisser exprimer ainsi l'être sensible tout en luttant contre la peur de la page blanche et en motivant leur créativité.

Le choix du blog convient parfaitement à notre activité par l'étymologie même du terme puisque rappelons que le terme blog provient de la contraction des mots anglais « web » et « log ». Ce dernier se traduit en français par « journal de bord », le journal qu'aurait rédigé l'apprenant durant son cursus universitaire. Ce nouveau support qui nous permet de placer nos écrits correspond donc parfaitement au thème de l'atelier:«Le sort d'une innovation dépend fortement du sens que lui donnent les acteurs, surtout quand il s'agit de changer leurs pratiques ${ }^{(5)}($ Brodin, p.6)

Concernant notre proposition d'écriture, il ne s'agit plus d'un voyage à travers l'espace mais dans le temps : l'apprenant raconte un événement qui s'est déroulé à l'ENS. Nous avons renouvelé le même atelier durant trois années successives car nous avons remarqué que le sujet intéresse particulièrement l'apprenant. La production concernerait un moment important de son vécu en tant qu'étudiant, un fait personnel qu'il partagerait avec ceux qu'il avait côtoyé pendant cinq ans. Le blog permet ce partage tellement important dans ce type de sujet: relater un événement personnel que l'on souhaite partager avec ses camarades.

Pour commencer, lors de la première séance, nous nous sommes convenus ensemble des différentes étapes du projet qui devait permettre à l'apprenant de nourrir sa volonté d'apprendre :

- Le contenu de l'activité est discuté ensemble en fonction des objectifs à atteindre ;

- Les caractéristiques du «Carnet de voyage» sont clairement définies ;

- Les différents moments de l'évaluation sont précisés ainsi que la forme que prendra l'évaluation ${ }^{(6)}$; 
Comme toute activité nouvelle, l'apprenant doit être correctement informé de l'activité d'écriture dans son ensemble et prendre ainsi conscience de l'enjeu de ce projet :

Les principaux acteurs d'une relation didactique, le plus souvent des enseignants et des élèves, sont réunis dans l'objectif de réaliser une activité à propos d'un contenu identifié. Cette activité est préalablement définie et préparée. Elle est finalisée et détermine le déroulement de l'ensemble de la relation didactique. (Jonnaert, Vander Borght, 2003, p.127)

$\mathrm{Au}$ cours de la première séance de l'activité, nous avons donc établi le projet sans pour autant le limiter à de la théorie ou à de la méthodologie. Il s'agissait surtout d'un changement de perspective sur notre manière de transmettre un savoir et sur notre manière d'écrire.Deux points caractérisaient ce projet :

- La confiance : indispensable pour que ce projet fonctionne ;

- La créativité : l'improvisation dans l'écrit.

Nous n'avons pas imposé un nombre minimum ou maximum de mots par production. Mais en général, les productions n'excédaient pas les 500 mots. Par ailleurs, nous avons, par ce travail de recherche, collecté auprès de nos apprenants différentes données à plusieurs moments du projet (questionnaire d'analyse des besoins, analyse des productions des apprenants, synthèse de fin d'activité...).

\section{QUAND LE « JE RÉDIGE » DEVIENT « J'ÉCRIS NUMÉRIQUE »}

Avec le module de production écrite, la théorie et la pratique sont étroitement liées. L'écriture numérique permet une activité pratique sous forme variée de ce qui a été appris précédemment. L'apprenant est motivé et participe activement à cet exercice nouveau notamment par l'utilisation du numérique. C'est un projet qui le conduit à s'investir avec facilité et intérêt (valeur didactique du projet). Dans un premier temps, les apprenants envoient leurs textes sur une adresse électronique. Nous leur avons demandé de mentionner leur nom et leur prénom dans l'objet (si le nom ne figure pas dans l'objet, le texte ne serait pas lu car les productions ne doivent pas être anonymes). Au fur et à mesure que les productions ont été envoyées, nous avons procédé à une première évaluation et nous les avons publiés sur le blog dans l'ordre de l'envoi des textes. Les apprenants lisent la production de leurs camarades en consultant le blog. En classe, nous partageons les textes et nous suggérons à l'auteur des modifications d'ordre syntaxiques, lexicales... Le fait que la production soit placée sur le blog, et que nous lui demandions son consentement pour certaines modifications ${ }^{(7)}$ de son texte, cela permet à l'apprenant d'acquérir une certaine confiance en soi, ce qui est primordial dans tout processus d'apprentissage : « un enseignant ne doit pas seulement s'efforcer de rendre un travail intéressant, mais il doit également 
envisager des moyens pour aider les élèves à avoir confiance dans leur capacité à réussir ». (Ardois, 2004, p. 46) Cette activité favorise une méthode de travail nouvelle pour nos apprenants notamment par l'ouverture vers le travail de l'autre en découvrant des productions aux formes variées. En plaçant les productions sur le blog, leurs écrits sont destinés à être accessibles et visibles par leurs camarades ainsi que par des apprenants appartenant à d'autres groupes.Ainsi, leurs productions sont valorisées lors de la mise en commun car elles acquièrent un statut public.

Une seconde évaluation a lieu au moment de la mise en commun en classe. Lors du partage des textes, l'apprenant peut modifier sa production. L'apprenant devait être ouvert, capable de s'adapter. Il accepte donc les propositions des autres : il écoute les remarques de ses camarades. Une mise en commun qui va jusqu'à parfois exclure l'enseignant de l'échange qui a lieu entre les apprenants. Nous avons d'ailleurs particulièrement apprécié la qualité des échanges lors de la mise en commun des écrits. Les scripteurs se sont à leur tour intéressés aux productions des autres, ce qui a permis un vrai travail d'échange. Ce projet les a soudés grâce au partage d'un vécu commun (des récits qui racontent des événements qui les concernent de près : leur vie d'apprenants). À travers la lecture des productions, ils trouvaient des éléments de vie communs. D'ailleurs, la communication continue entre eux, jusqu'en dehors des horaires des cours puisqu'ils commentent entre eux des productions placées sur le blog parfois avant même que le cours ait lieu.

Certains affirment avoir rédigé après avoir consulté sur le blog les productions mises en ligne. C'est pourquoi, nous avons accepté à ce que les apprenants, confrontés à la page blanche, puissent lire la production de leur camarade avant de rédiger leur propre texte. L'apprenant produit à son rythme et choisit librement le moment le plus adéquat pour rédiger.

Certains font des choses très vite, $d$ 'autres très lentement. Tout enseignant ou formateur en fait l'expérience quotidiennement. Dans le domaine de l'apprentissage, ces différences dites de nature qualitative ont trait à la vitesse à laquelle un apprentissage peut être effectué et au niveau de maitrise qui peut être atteint. (Lautrey, 2006, p. 83)

L'enseignant ne peut exiger de l'apprenant d'être créatif dans une salle de cours et en 1h30. Encore faut-il être inspiré au moment où l'enseignant propose le sujet de production. La créativité ne peut être un exercice purement improvisé. L'environnement de travail change puisque la rédaction s'effectue en dehors des heures de cours et si l'apprenant le souhaite en dehors de l'ENS. L'apprenant n'est plus limité par le temps ni par l'espace, ce qui est un grand atout lorsqu'on rédige et particulièrement lorsqu'il s'agit d'une langue étrangère. ${ }^{(8)}$ Ainsi, le temps alloué à l'écriture n'est plus limité. Par ailleurs, l'utilisation de l'écran 
aide l'apprenant à mieux s'exprimer car il échappe à l'urgence de l'écriture en classe. La créativité se développe dans les espaces hors pression.

Ce type d'exercice permet de ne pas interrompre l'écriture. Nous avons proposé à l'apprenant d'autres sujets pour qu'il puisse continuer à écrire pendant la période de son stage dans les établissements scolaires. ${ }^{(9)}$ Les productions rédigées pendant leur stage sont affichées sur le blog créant ainsi des interactions horizontales entre les participants. En revanche, cet exercice n'a ni été évalué, ni fait l'objet d'une mise en commun à leur retour de stage. Les apprenants les ont consultés individuellement afin de lire les productions de leurs camarades. Ils ont produits à partir de propositions clairement définies avant leur départ en stage. Pour les apprenants en FLE, cette pratique leur donne l'accès à une autonomie et à une motivation renforcée.

Le fait que les textes soient placés sur le blog réduit de manière significative le temps de l'apprentissage car l'ensemble du groupe a déjà pris connaissance des textes et le travail sur les productions est plus rapide et plus fructueux. Nous nous sommes rendu compte qu'au fil des semaines, les apprenants ont pris goût à la lecture mutuelle de leurs écrits.

Nous vous proposons deux extraits de productions qui illustrent l'utilisation de l'écriture numérique chez l'apprenant en FLE :

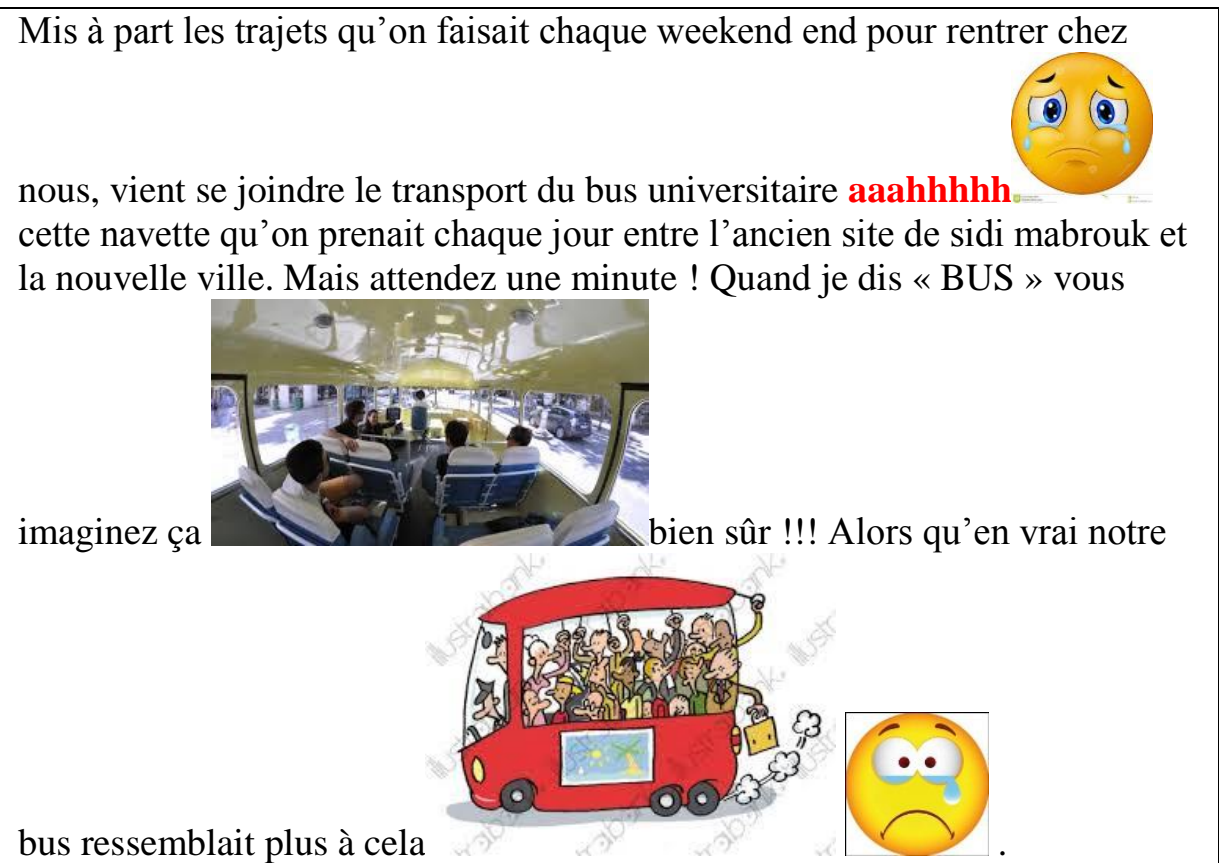




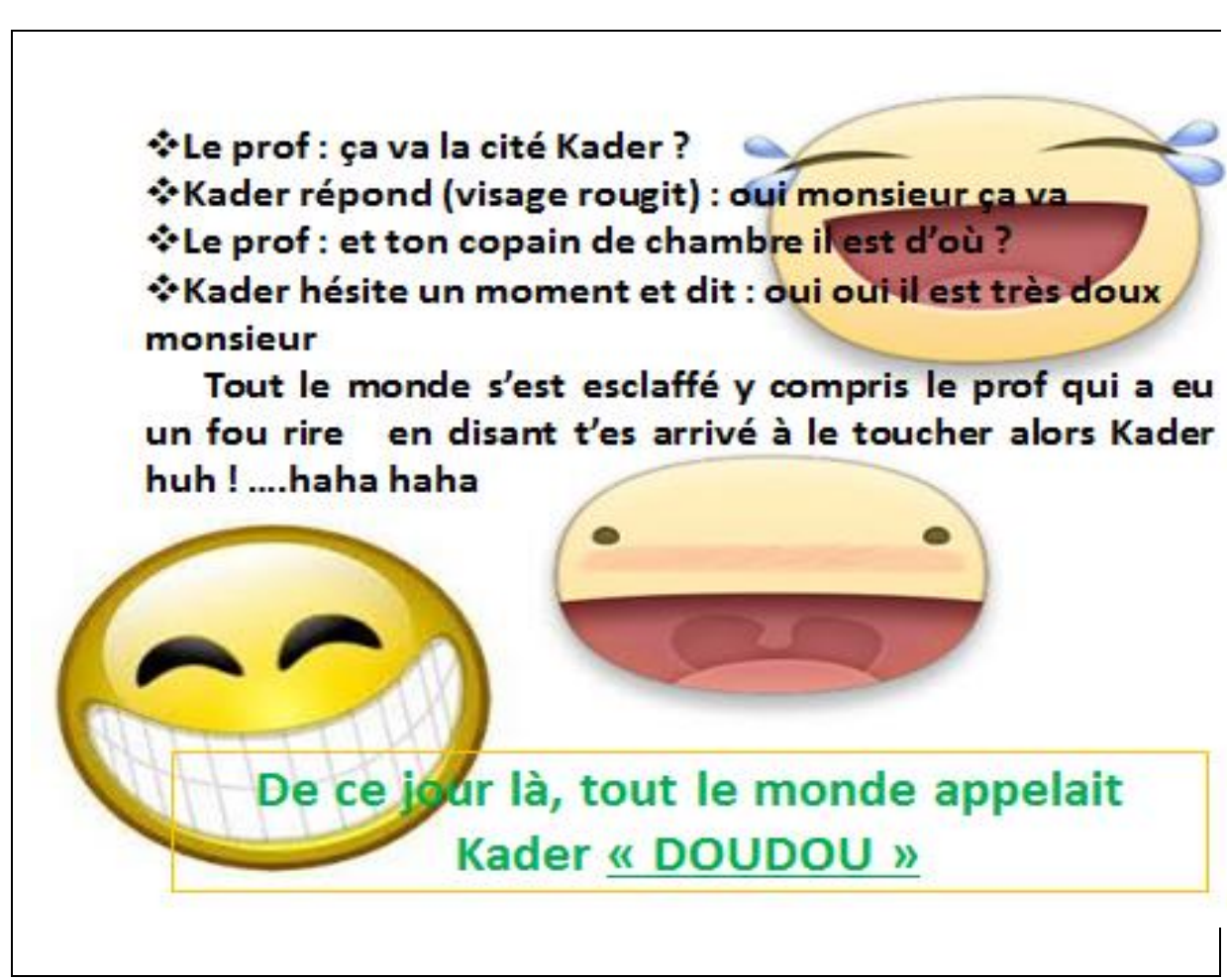

Il est important que l'enseignant participe également à ce type d'activité en publiant également sur le blog un texte. Dans l'activité « Carnet de voyage », l'enseignant est invité à écrire sur un événement qui a eu lieu durant sa carrière professionnelle et de préférence durant les cinq dernières années afin que la période du cursus universitaire des Bac +5 soit respectée. La participation de l'enseignant permet également d'améliorer la relation entre l'enseignant et les apprenants. Ce type de sujet favorise l'utilisation de l'humour, de la fantaisie et permet aux apprenants d'employer leur créativité en insérant des éléments qui sortent de l'ordinaire. La créativité et l'humour ont traduit la part de subjectivité dans l'écrit. Les émotions de chaque apprenant jouent un rôle essentiel dans l'écriture.

\section{L'ÉVALUATION}

Le choix d'utiliser l'écriture numérique pour les productions des apprenants, nous conduit à réfléchir sur la difficulté d'intégrer la créativité au sein du processus d'évaluation. En effet, la créativité relève de la subjectivité alors que l'évaluation se veut avant tout objective. Il faudrait donc que la créativité et l'innovation soient conciliables avec les règles de l'évaluation. Comment pouvons-nous par conséquent évaluer la créativité ? L'activité «Carnet de voyage » n'est pas le seul exercice évalué au cours du semestre. Les 
apprenants continuent de produire sur papier, l'évaluation semestrielle est établie en fonction de l'ensemble des productions du semestre. Nous avons donc opté pour une évaluation continue. Les apprenants qui écrivent numérique doivent être responsable car il est difficile pour l'enseignant de vérifier que c'est bien l'apprenant en question qui a rédigé le texte.

L'évaluation s'effectue à deux niveaux. Dans un premier temps, nous utilisons les mêmes critères que pour une production écrite sur papier, les apprenants sont évalués sur des points normatifs. Ils doivent valider les compétences exigées par les programmes suivis par l'ENS. Le projet doit donc respecter les exigences normatives :

- Syntaxe des phrases correcte ;

- Respect de la concordance des temps et des modes ;

- Utilisation des connecteurs logiques qui structurent le texte ;

- Le vocabulaire est varié, précis et adapté au sujet.

Puis dans un second temps, nous évaluons l'apprenant sur sa créativité par l'emploi des outils du numérique :

- Respect de la procédure de travail

- Travail sur la forme du texte (taille des caractères, police, gras, italique, souligné, couleur...)

- L'intégration d'émoticônes, de dessins, d'images...

Ce projet ne supprime pas les anciennes normes mais il met en place de nouvelles normes dans la pratique d'enseignement et d'évaluation.

\section{Les étapes de l'évaluation}

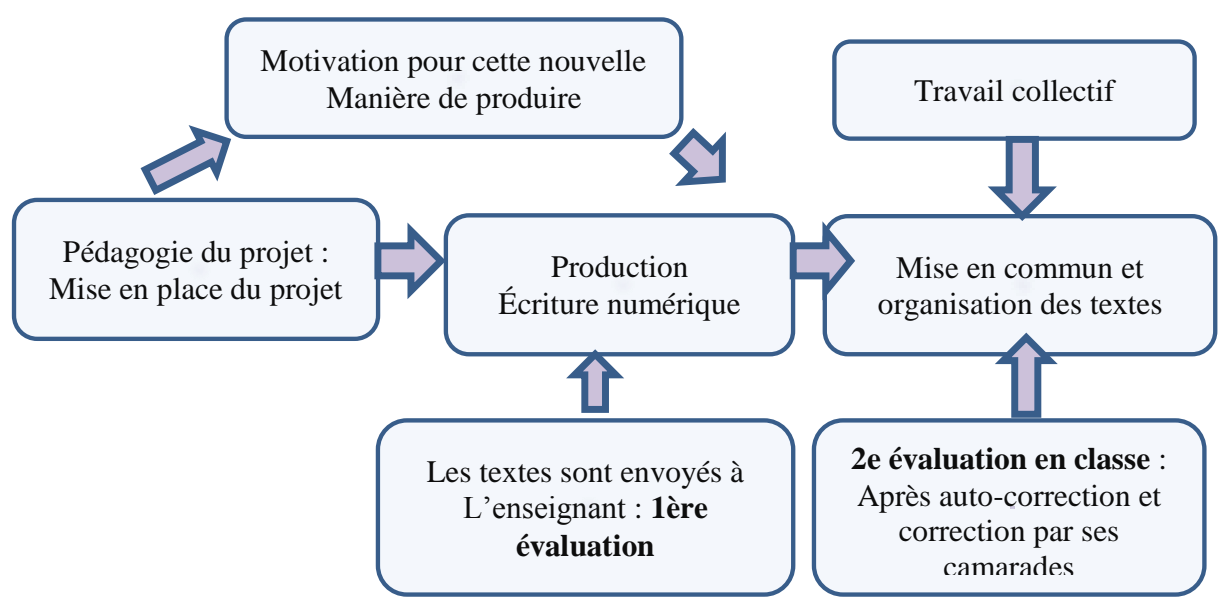


En aucun cas les productions doivent être comparées lors de l'activité, particulièrement lors de la mise en commun des textes en classe. Le partage des textes permet l'auto-correction par l'apprenant et par ses camarades qui reconsidèrent l'erreur et tout ce qu'elle représente puisqu'elle permet de diminuer la pression évaluative.

... l'évaluation (...) consiste à examiner l'adéquation des résultats obtenus au terme de la réalisation de l'action d'éducation ou de formation, par rapport aux résultats attendus (c'est-à-dire, par rapport aux objectifs de l'action d'éducation ou de formation) (Roegiers, 2007, p. 57)

Il faut être prudent à ce que la mise en commun des productions n'entraîne pas une comparaison entre les apprenants mais une évaluation critériée individuelle effectuée par l'enseignant :

L'évaluation est critériée lorsqu'elle ne compare pas les élèves entre eux, mais qu'elle détermine, par la référence à des critères, si l'élève est capable de passer à d'autres apprentissages. (Tagliante, 2005, p. 9)

L'évaluation ne se situe pas seulement à la fin de l'activité mais en deux temps pour permettre à l'apprenant d'améliorer son travail et procéder à des ajustements et une réflexion sur la pratique de l'écrit. Il accepte les remarques et les suggestions formulées par ses camarades. Il doit parvenir à maîtriser les codes linguistiques et culturels français pour écrire une anecdote émanant de son quotidien et le conduire progressivement à s'auto-évaluer. Ce travail de réécriture permet à l'apprenant d'acquérir une motivation interne qui lui permettra de continuer à écrire, en particulier après la formation.

\section{AVIS DES APPRENANTS CONCERNANT CETTE FORME D'ÉCRITURE}

Nous avons demandé aux apprenants d'expliciter les apprentissages qu'ils ont réalisés lors de cet enseignement par rapport à ce qu'ils attendaient de ce projet par rapport à un cours traditionnel, ce que ce cours leur a apporté etce qui leur a manqué.Le plus grand nombre de participants affirment avoir apprécié cette nouvelle forme d'écriture. Ils ont pu produire en dehors des heures de cours et à l'extérieur de l'ENS et éviter ainsi le stress inutile lié à des contraintes particulièrement à la limitation de temps. Ils ont su utiliser leurs ressources créatives, souvent inemployées, pour une nouvelle façon d'écrire. Ils ont été en mesure d'évaluer eux-mêmes leurs écrits et surtout à s'auto-corriger. Grâce à ce partage de textes, ils ont compris que les erreurs font partie du processus d'apprentissage. L'écriture numérique leur a permis de réaliser une œuvre commune tout en appliquant l'ensemble de leurs connaissances. Ils ont affirmé être curieux de voir ce que leurs camarades avait écrit en consultant le blog. Ils 
ont apprécié pouvoir contribuer à l'amélioration des productions de l'ensemble du groupe. C'est grâce au regard porté par leurs camarades sur leurs écrits qu'ils ont pu établir une certaine distanciation critique par rapport à leur écrit.

\section{CONCLUSION :}

Cet article présente le travail effectué auprès d'apprenants (Bac $+5:$ futurs enseignants du secondaire) au cours d'une activité de production écrite intégrant l'écriture numérique.

Nous retenons de notre recherche la manière dont les apprenants s'approprient les potentialités créatives de cette nouvelle pratique d'écriture. Elle leur a permis de mieux apprendre en donnant du sens à l'apprentissage. Ce projet, visant l'autonomie de l'apprenant, nous a amené ensemble à concevoir ensemble cette activité :

- Co-créer un ensemble d'anecdotes

- Explorer, apprendre, interagir en groupe

Le fait que la production soit placée sur le blog et qu'elle fasse l'objet d'une lecture en classe donne le sentiment à l'apprenant que son travail a de la valeur même s'il ne répond pas totalement aux objectifs initialement précisés. La valorisation de la production motive l'apprenant qui essaiera de bien faire même lorsque le travail n'est pas noté.

L'écriture numérique permet aux apprenants d'acquérir une motivation non plus seulement motivée par l'évaluation mais une volonté de continuer à écrire, en particulier après la fin de leur formation, lorsqu'ils deviendront à leur tour des enseignants.

Nous avons souhaité continuer l'écriture numérique avec les apprenants des deux promotions précédentes (2015-2016 et 2016-2017), devenus à présent de jeunes enseignants. Nous leur avons proposé de prolonger le «Carnet de voyage » : la vie professionnelle d'un jeune enseignant racontée à partir de leurs propres anecdotes. Nous avons reçu plusieurs productions mais beaucoup n'ont pas donné suite à l'aventure. Sans doute la pratique de l'écriture numérique aurait dû être initiée plus tôt dans le cursus universitaire de nos apprenants pour que cette activité devienne une habitude. Cette activité devrait être également encouragée auprès de nos collègues au sein d'autres modules du département de français. 
ANNEXES

Questionnaire réalisé auprès d'apprenants (nombre 46) niveau $\mathrm{Bac}+5$ (promotion 2016-2017). Les apprenants notent les éléments qui ont facilité l'acte d'écrire et/ou au contraire ceux qui ont plutôt freiné son avancement :

\section{ENVIRONNEMENT POUR ÉCRIRE}

\begin{tabular}{|l|c|c|c|}
\cline { 2 - 4 } \multicolumn{1}{c|}{} & Oui & Non & Indifférent \\
\hline $\begin{array}{l}\text { Être limité par le } \\
\text { temps }\end{array}$ & $26 \%$ & $68 \%$ & $6 \%$ \\
\hline $\begin{array}{l}\text { Être contraint de } \\
\text { rédiger dans un } \\
\text { lieu imposé }\end{array}$ & $23 \%$ & $67 \%$ & $10 \%$ \\
\hline $\begin{array}{l}\text { Préférer écrire } \\
\text { dans une salle de } \\
\text { classe }\end{array}$ & $7 \%$ & $81 \%$ & $12 \%$ \\
\hline $\begin{array}{l}\text { Préférer produire } \\
\text { à l'ENS }\end{array}$ & $57 \%$ & $39 \%$ & $4 \%$ \\
\hline
\end{tabular}

\section{L'ÉCRITURE COLLECTIVE}

\begin{tabular}{|c|c|c|c|c|}
\hline & & Oui & Non & Indifférent \\
\hline \multirow{2}{*}{ Supports } & $\begin{array}{l}\text { Textes } \\
\text { littéraires }\end{array}$ & $39 \%$ & $45 \%$ & $16 \%$ \\
\hline & $\begin{array}{l}\text { Productions des } \\
\text { apprenants }\end{array}$ & $72 \%$ & $23 \%$ & $5 \%$ \\
\hline \multicolumn{2}{|c|}{ Produire à partir d'un projet } & $56 \%$ & $41 \%$ & $3 \%$ \\
\hline \multicolumn{2}{|c|}{$\begin{array}{l}\text { La mise en commun des } \\
\text { productions }\end{array}$} & $86 \%$ & $12 \%$ & $2 \%$ \\
\hline
\end{tabular}




\section{BIBLIOGRAPHIE:}

Ardois, C. (2004). Former des apprenants responsables, Bruxelles : De Boeck \& Larcier s. a.

Brodin, É. Innovation en éducation et innovation dans l'enseignement des langues : quels invariants ?Université Paris 3, https://acedle.org/old/IMG/pdf/EB041122-2-2.pdf

Jonnaert, P., Vander B. (2003).Créer des conditions d'apprentissage, ( $2^{\mathrm{e}}$ édition). Bruxelles : De Boeck \& Larcier s. a.

Kavian, E. (2009).Écrire et faire écrire. Manuel pratique d'écriture, (2 édition). Bruxelles : Groupe De Boeck s.a.

Lautey, J. (2006). «Les différences de cheminement dans l'apprentissage », In Apprendre et faire apprendre, sous la direction de Etienne Bourgeois et Gaëtane Chapelle, Paris : Presses universitaires de France.

Lebrun, M. (2007).Théories et méthodes pédagogiques pour enseigner et apprendre. Quelle place pour les TIC dans l'éducation?(2 édition). Bruxelles : De Boeck \& Larcier s. a.

Roegiers, X. (2007). Analyser une action d'éducation ou de formation, Bruxelles : De Boeck \& Larcier s. a.

Tagliante, C. (2005). L'évaluation et le Cadre européen commun. Techniques et pratiques de classe, Paris, Clé international.

Références supplémentaires :

Chiss, J-L. (2012). L'écrit, la lecture et l'écriture. Théories et didactiques, Paris :L'Harmattan.

Lubart, T. (2005).Psychologie de la créativité, Paris : Armand Colin.

Mottier, L. (2015). Évaluations formative et certificative des apprentissages, Enjeux pour l'enseignement. Louvain-la-Neuve : De Boeck Éducation.

Narcy-Combes, J-P. (2005).Didactique des langues et TIC : vers une rechercheaction responsable, Paris : Éditions Ophyrys.

Roche, A. \&Guiguet, A. \&Voltz, N. (2011).L'atelier d'écriture. Éléments pour la rédaction du texte littéraire, ( $3^{\mathrm{e}}$ édition). Paris : Armand Colin. 


\section{Notes :}

(1)-Les textes sont publiés dans l'ordre où ils ont été envoyés quel que soit le contenu (anecdote triste, gaie) pour rester fidèle à la réalité, c'est-à-dire comme elles sont apparues dans la vie réelle.

(2)-Bac+5, il s'agit de futurs enseignants au lycée.

(3)-Nous notons que la seconde édition de l'ouvrage d'Eva Kavian, Écrire et faire écrire. Manuel pratique d'écriture est enrichi d'une webographie des ateliers d'écriture.

(4)-Les aides à l'écriture d'Eva Kavian : « un fragment de conversation, une rencontre, dessiner une fenêtre et écrire quelques lignes, relever un instant ténu, anodin, coller un papier, un ticket et écrire quelques lignes, quelques lignes sur les odeurs, une perte, un texte écrit la nuit, un sentiment de solitude, une liste ( de n'importe quoi) et deux lignes de commentaires, une décision, une étape du trajet, dessiner un morceau de plan, une adresse et écrire quelques lignes, un souvenir ranimé par une vision, un rêve, quelques notes de lecture, une citation, quelques lignes sur le silence, le personnage écrit ce qu'il voit...»

(5)-L'auteur souligne.

(6)-La proposition d'écriture ainsi que les moments de l'évaluation ont été clairement établis en classe lors de la première séance. Cette étape primordiale permet l'activation des connaissances acquises tout au long de leur cursus universitaire.

(7)-Il s'agit d'aides mutuelles entre les apprenants pour reformuler certains passages de la production.

(9)-Les habitudes de chaque apprenant sont respectées : le lieu et le moment sont librement choisis par l'apprenant.

(10)-Pendant trois semaines les apprenants sont orientés dans des établissements du primaire ou du secondaire. Durant cette période les cours à l'ENS sont suspendus. 